\title{
Observation of coherent Smith-Purcell radiation using an initially continuous flat beam
}

\author{
J. Gardelle, L. Courtois, and P. Modin \\ CEA, CESTA, F-33114 Le Barp, France \\ J. T. Donohue \\ Centre d'Etudes Nucléaires de Bordeaux-Gradignan, BP 120, 33175 Gradignan, France
}

(Received 15 April 2009; published 9 November 2009)

\begin{abstract}
A few years ago a new theory for producing coherent Smith-Purcell (SP) radiation from an initially continuous beam was proposed. This experiment confirms that two-dimensional theory. The beam was typically $10 \mathrm{~cm}$ wide, a few mm thick, with a peak current of $200 \mathrm{~A}$ and beam energy of $85 \mathrm{keV}$. The $10 \mathrm{~cm}$-wide grating had twenty 2 -cm periods, and radiation was produced at the fundamental frequency near $4.5 \mathrm{GHz}$. Second and third harmonics were observed at the expected angles. Beam bunching was measured using a B-dot probe placed at the end of a groove. Generally good agreement between this experiment and the $2 \mathrm{D}$ theory is found.
\end{abstract}

DOI: 10.1103/PhysRevSTAB.12.110701

PACS numbers: 41.60.Cr, 52.65.Rr

\section{INTRODUCTION}

In 1953 Smith and Purcell (SP) [1] sent a $300 \mathrm{keV}$ electron beam along a diffraction grating, and observed light that satisfied the condition, $\lambda=L(1 / \beta-\cos \theta) /|n|$, where $\lambda$ denotes the wavelength of radiation produced at angle $\theta$ with respect to the beam, the period is $L$, and $n$ is the order. Here $\beta=v / c$ is the usual relative velocity. Since its discovery, SP radiation has been the subject of much theoretical and experimental work, both as a tunable source for radiation and as a diagnostic tool for electron beams. Experiments have produced SP radiation using beams of energy ranging from a few $\mathrm{keV}$ to $28 \mathrm{GeV}$, with both continuous and prebunched beams [2-9]. Some relevant theoretical work was performed by Liu and Tripathi [10] and a lengthy treatise has been written by Shestopalov [11]. It was realized long ago that if a bunch of electrons was smaller than $\lambda$, the energy emitted would scale as $N_{e}^{2}$, where $N_{e}$ is the number of electrons per bunch. This is called intrabunch coherence, in which the electromagnetic field is emitted collectively by all electrons in the bunch. If the bunch is part of a periodic train, the frequencies emitted must be integer multiples of the bunching frequency. It then follows that the radiation can occur only at certain angles, provided the bunching frequency exceeds the SP threshold, $f_{\min }=c / L(1+1 / \beta)$. Even if this does not hold, there may be harmonic components in the current for which the condition is satisfied, and then one expects copious monochromatic radiation at a welldefined angle with respect to the beam. This has been called interbunch coherence by Andrews and Brau [12] (hereafter $\mathrm{AB}$ ), who have proposed a new model for coherent SP radiation. In two subsequent articles [13] they, with their collaborators, developed a detailed model for bunching an initially continuous beam passing over a lamellar grating, and the subsequent emission of radiation. Using Floquet theory, $\mathrm{AB}$ establish the dispersion relation that relates the frequency $\omega$ and the axial wave number $k$ of the evanescent wave that the grating supports. The operating point of the system is determined by the intersection of the dispersion relation with the beam line, $\omega=k v$. In its neighborhood an instability of the coupled system occurs, leading to exponential growth of beam bunching and wave amplitude. For low energy, the intersection occurs on that part of the dispersion relation where $d \omega / d k$ is negative. If so, the system behaves like a backward-wave oscillator (BWO). The resulting bunching is at frequency $\omega$, but this frequency is always less than the minimum allowed SP frequency, provided the intersection occurs in the lowest Brillouin zone. However, if the bunching is strong enough, harmonics of this frequency will be present in the current, and these may be allowed SP frequencies. Then interbunch coherent SP radiation will be emitted at the SP angle. According to this model, tunable coherent radiation may be generated by a compact source, since energies of several tens of $\mathrm{keV}$ are sufficient.

The $\mathrm{AB}$ analysis is $2 \mathrm{D}$, i.e., the beam and grating are supposed to extend infinitely in the direction of the grooves. To test the model, two of us performed a 2D simulation [14] using the commercially available particle-in-cell (PIC) code MAGIC [15]. It was found that the simulation supported all the model's predictions. The energy was such that BWO behavior was seen, with wave energy flowing upstream. The evanescent nature of the wave in the direction perpendicular to the grating was established, and the e-folding distance is given by $\gamma \beta \lambda / 2 \pi$, where $\gamma=\left(1-\beta^{2}\right)^{-1 / 2}$. For the parameters of the simulation, this was about $7 \mathrm{~mm}$. Thus, the beam must pass within this distance from the grating in order to interact strongly with the wave. When a beam of thickness $5 \mathrm{~mm}$ passing $2 \mathrm{~mm}$ above the grating was included, bunching at the expected frequency was observed, provided the current was $>10 \mathrm{~A} / \mathrm{m}$. For currents much 
greater than that, the expected $I^{1 / 3}$ behavior was found for $\operatorname{Im} \omega$, the temporal gain. Monochromatic radiation at the second harmonic of the bunching frequency was produced at the SP angle. The Floquet nature of the evanescent wave was confirmed by the presence of many wave numbers in the spatial fast-Fourier-transforms (FFTs). The simulation thus provided considerable support for the $\mathrm{AB}$ model. However, it was also found that upon reaching the ends of the grating the evanescent wave was partially reflected, and partially emitted as free radiation at the fundamental frequency. Copious emission occurred at both ends in all directions.

$\mathrm{Li}$ and collaborators [16] also provided support for the $\mathrm{AB}$ model using the PIC code MAGIC. A different 2D model was proposed by Kumar and Kim [17], in which the beam was taken to be a sheet of zero thickness, unlike the uniform plasma of the $A B$ model. However, their results were in good agreement with those of $\mathrm{AB}$, except that they suggested extracting the evanescent wave as free radiation. Thus theory and simulations appear to confirm the $\mathrm{AB}$ paradigm, and open the way to compact tunable $\mathrm{SP}$ lasers. It is therefore of great interest to confirm this theory experimentally. Of course, no experiment can be performed in two dimensions, but it seems reasonable to suppose that the 2D theory would be valid if both the beam and the grating were large compared to the wavelength of the evanescent wave. The present experiment was designed to demonstrate the essential validity of the 2D theory. For this demonstration experiment, we worked in the range of microwave frequencies, as in our simulation, because the requirements of beam quality were not extremely difficult. In addition, equipment for the measurement was available, as well as a reliable pulsed-power source. Since the AB theory applies at all scales, a suitably scaled version of our experiment could be expected to generate radiation at any wavelength. However, the requirements on the flat wide beam might impose practical limits, because at a much smaller scale, the very thin beam would have to pass quite close to the grating.

Although the $2 \mathrm{D}$ theory had not been thoroughly validated, an important experiment was reported at the 2008 FEL Conference by Andrews and coworkers, and subsequently published in the present journal [18]. This experiment in the THz regime observed the evanescent wave in a grating equipped with sidewalls. In this case, as the authors show, the predictions of the 2D theory differ substantially from the 3D theory that had been presented earlier by Andrews, Brau, and Jarvis [19]. The experiment, using a narrow beam, confirms the latter, at least for the evanescent wave. However, the second harmonic is seen weakly, at best, and the incoherent SP signal is of comparable intensity to that of the evanescent wave.

A simple explanation for the fact that no existing experiments have validated the 2D theory is that it may not apply to experiments with narrow beams of cylindrical cross section. A second possibility is that none of the earlier experiments used a current sufficiently intense to achieve gain. In addition to Ref. [19], another 3D theory of coherent SP radiation has been proposed by Kim and Kumar [20]. Both groups find that a scenario similar to the 2D theory should be valid, although the details may be quite different. In particular, the evanescent wave is expected to be considerably wider than the beam, provided the grating is wide enough to support it. Two of us have presented a 3D MAGIC simulation, which indicates that for our grating (which has open-ended grooves) using a flat beam of equal width, results quite similar to the 2D scenario are expected [21].

\section{DESCRIPTION OF THE EXPERIMENT}

To test the $\mathrm{AB}$ model, we performed an experiment based on the simulations reported in Refs. [14,21]. In order to have a system in which the $2 \mathrm{D}$ approximation may be valid, an electron beam $10 \mathrm{~cm}$ wide and a few mm thick is provided by a high-current pulsed-power generator operating in single-shot mode. The power supply, built at CESTA, can furnish $1 \mathrm{kA}$ at maximum voltage, of $200 \mathrm{kV} \pm 5 \%$, for a pulse of $300 \mathrm{~ns}$ FWHM. Its maximum repetition rate is $100 \mathrm{~Hz}$. The parameters of our setup are given in Table I, and its photograph is shown in Fig. 1(a). The mirror may be moved along the grating and its angle may be adjusted to reflect the SP radiation into the forward direction. The setup is enclosed in a cylindrical vacuum chamber, which is surrounded by a long pulsed solenoid that provides a uniform axial magnetic field. The waveforms of the applied voltage and the current at the beam dump are displayed in Fig. 1(b). A time-integrated image of the beam at the collector on thermosensitive paper may be seen in Fig. 1(c). The result of a 3D MAGIC simulation is also shown for comparison, but this represents an instantaneous rather than an integrated image. The physical effect responsible for the shape of the beam is the wellknown $\vec{E} \times \vec{B}$ drift [22]. The magnetic field is directed along the negative $z$ direction (opposite to the beam). In the middle, the electric field is mainly in the positive $y$ direction, and this produces a drift of the beam in the negative $x$ direction. At the extremities, however, the electric field also has a significant $x$ component, directed towards the beam. This component leads to a vertical drift, upwards at one end and downwards at the other. All of these effects are clearly present, and the agreement between measurement and simulation is quite satisfactory. The voltage is measured with a capacitive sensor, while the current is measured by a Rogowski coil placed underneath the grating on the current return stalk. As may be seen in Fig. 1(a), a B-dot probe may be placed at the extremity of any groove in the grating, where it measures the time derivative of the magnetic flux in the $x$ direction. The radiation emerges from a plexiglas window at the downstream end of the solenoid, and two horn antennas are used 
TABLE I. Experimental parameters.

\begin{tabular}{lc}
\hline \hline Parameters & Value \\
\hline Applied voltage & $(85 \pm 5) \mathrm{kV}$ \\
Current & $(180 \pm 30) \mathrm{A}$ \\
Current pulse duration (FWHM) & $300 \mathrm{~ns}$ \\
Beam thickness & $2 \mathrm{~mm}$ \\
Beam-grating distance & $2 \mathrm{~mm}$ \\
Beam width & $10 \mathrm{~cm}$ \\
Grating period & $2 \mathrm{~cm}$ \\
Grating groove depth & $1 \mathrm{~cm}$ \\
Grating groove width & $1 \mathrm{~cm}$ \\
Grating width & $10 \mathrm{~cm}$ \\
Number of periods & 20 \\
External magnetic field & Variable from 0 to $1 \mathrm{~T}$ \\
\hline \hline
\end{tabular}

to detect the evanescent wave (S-band) and SP radiation at its second and third harmonics (X-band). The antenna signals are transmitted to a fast oscilloscope of bandwidth $12 \mathrm{GHz}$. These antennas may be moved so as to obtain the antenna pattern of the device at both the evanescent and harmonic frequencies. An experiment with a setup similar to that shown here was performed by Skrynnik, Korneyenkov, and Demchenko [23]. They used a very low energy sheet beam (2.5-5 keV) and reported observing SP radiation at harmonics of the evanescent wave.

\section{RESULTS}

According to the $\mathrm{AB} 2 \mathrm{D}$ theory, the frequency of the evanescent wave for beam energy $85 \mathrm{keV}$ is $4.56 \mathrm{GHz}$. This is below the SP threshold of $5.17 \mathrm{GHz}$, but the second harmonic at $9.12 \mathrm{GHz}$ is SP allowed. It should be emitted from the grating at $\theta=75^{\circ}$. If the mirror is inclined at half this angle, the SP radiation should emerge from the vacuum chamber in the forward direction. The third harmonic is expected at $36^{\circ}$. Since our beam energy varies during the pulse, the intersection of the beam line with the dispersion relation moves, causing a frequency shift in the radiation. In Fig. 2 we show the measured time signals of a shot, as detected by the X-band horn (a), the S-band horn (b), and the B-dot probe placed at the end of a groove (c). The corresponding FFTs are shown on the right. The signal shown in Fig. 2(a) is steady in time, and its FFT indicates the second harmonic, plus a smaller contribution at the third harmonic. In contrast, the time signals of 2(b) and 2(c), which are dominated by the evanescent wave at 4.3 GHz, are more chaotic, although both FFTs indicate some second and third harmonics.

The signal shown in Fig. 2(c) may be converted, using the measured properties of the probe, to a measurement of the peak magnetic field at the frequency of the evanescent wave. The result is $8 \pm 1 \mathrm{G}$. Since the probe is placed about $1 \mathrm{~cm}$ beyond the open end of the groove, it measures less than the field in the groove. In our MAGIC 3D simulation, the predicted value is $10 \mathrm{G}$ at the probe's position, and $35 \mathrm{G}$ in the center of the groove. The former is consistent with our direct measurement.

As we noted above, the beam energy varies over the duration of the pulse, shifting the frequency of the SP radiation. To illustrate this we show in Fig. 3(a) a portion of the sliding FFT, as measured by the X-band horn. The frequency decreases slowly from a maximum early in the pulse. This decrease is consistent with the hypothesis that the device functions like a backward-wave oscillator. If the intersection occurred on the rising part of the dispersion relation, a decrease in energy would cause an increase in frequency. In fact, this frequency shift may prove of some
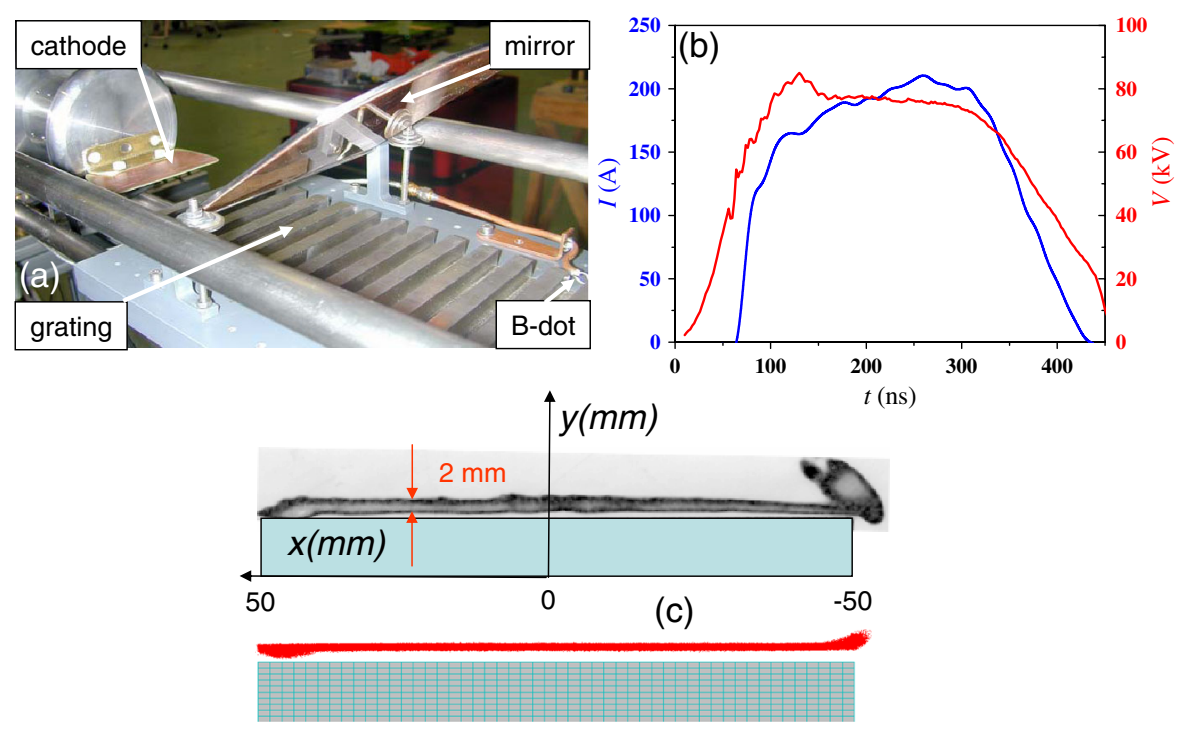

FIG. 1. (Color) (a) Photograph of the setup. (b) Typical voltage and current pulse forms. (c) Imprint of beam on thermosensitive paper at beam stop. Also is shown a MAGIC 3D simulation of section of beam at stop. 

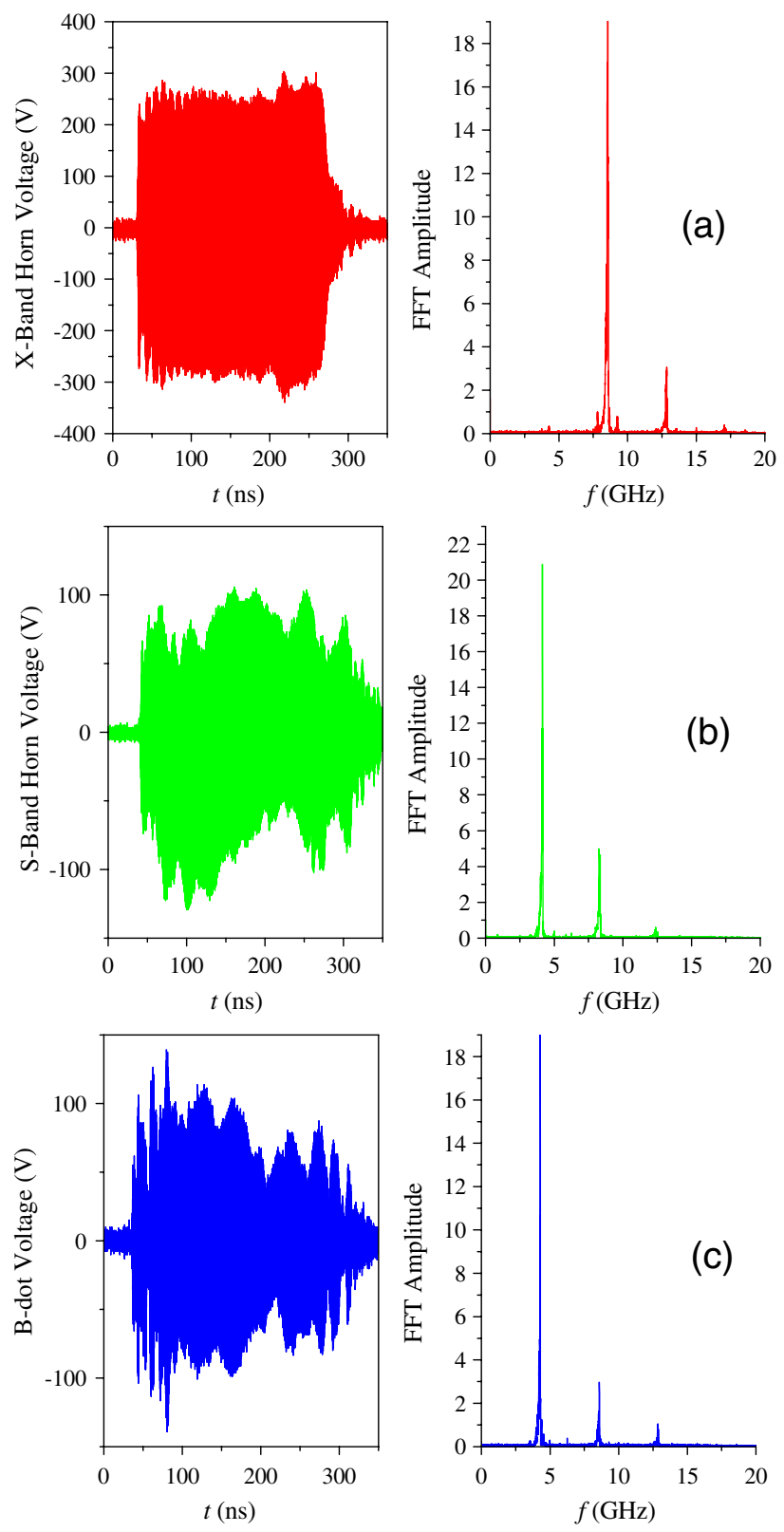

FIG. 2. (Color) Time signals and their FFTs: (a) X-band horn; (b) S-band horn for evanescent wave; (c) magnetic field (parallel to groove) at a groove end. interest as energy diagnostic for intense electron beams. In Fig. 3(b) we show the frequency as a function of time (lefthand scale), along with the beam kinetic energy deduced from it, assuming the $\mathrm{AB}$ dispersion relation (right-hand scale). The energy thus deduced is less than the voltage measured in the generator. We attribute this discrepancy to the space-charge depression produced by the intense beam, and to the uncertainty in the voltage measurement.

Parametric variations were performed to investigate the behavior of the setup. One was to vary the maximum voltage, and observe the shift in the main peak of the FFT for the X-band horn. This is shown in Fig. 4(a), where the frequency is shown as a function of peak applied voltage. A second example is shown in Fig. 4(b), where the energy/area collected in the X-band horn is shown using a logarithmic scale for six different distances $d_{\mathrm{AK}}$ (the vertical distance of the cathode above the grating). The signal is seen to decrease slowly for small $d_{\mathrm{AK}}$, and then more rapidly as the height approaches the theoretical efolding length of $7 \mathrm{~mm}$. However, it is clearly not a simple exponential. From this, we conclude that it is essential for $d_{\mathrm{AK}}$ to be of order $2 \mathrm{~mm}$ in order to reach high intensity. Finally, the width of the beam was reduced by choosing a narrower cathode. Even with a beam width of $2 \mathrm{~cm}$, a smaller but still substantial signal was obtained. Additional variations involved displacing the mirror and varying its angle. The latter enabled us to scan the angular distribution of radiation emitted in the forward direction. In particular, we could observe separately the second and third harmonics at the appropriate angles. We found that placing the mirror near the upstream end of the grating gave the highest output. When the mirror was removed, signals were still observed, but at considerably lower levels. We also varied the axial magnetic field, finding that fields $\geq 0.4 \mathrm{~T}$ were sufficient to obtain optimal signals.

The antenna pattern of the device was measured by displacing the horns along vertical and horizontal arcs at a fixed distance of $2.5 \mathrm{~m}$ from the exit of the vacuum chamber. By fitting these data with Gaussian functions for the horizontal and vertical angles, and assuming the width in the polar angle $\theta$ to vary linearly in the azimuth $\varphi$, we get an estimate of the energy radiated $d E / d \Omega$ near the
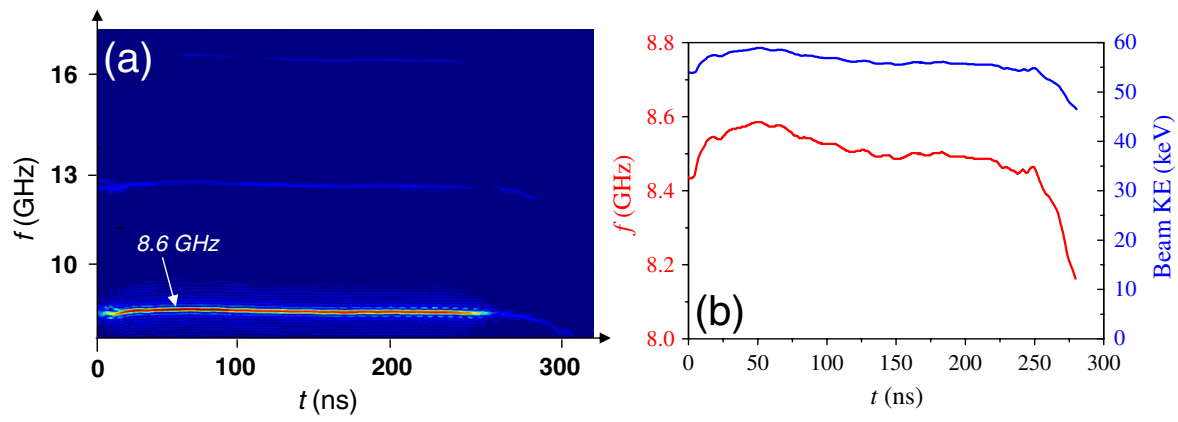

FIG. 3. (Color) (a) Sliding Fourier transform showing time variation of frequency of X-band horn signal. (b) Second harmonic frequency vs time (left-hand scale) and kinetic energy of electrons as deduced from the AB dispersion relation (right-hand scale). 

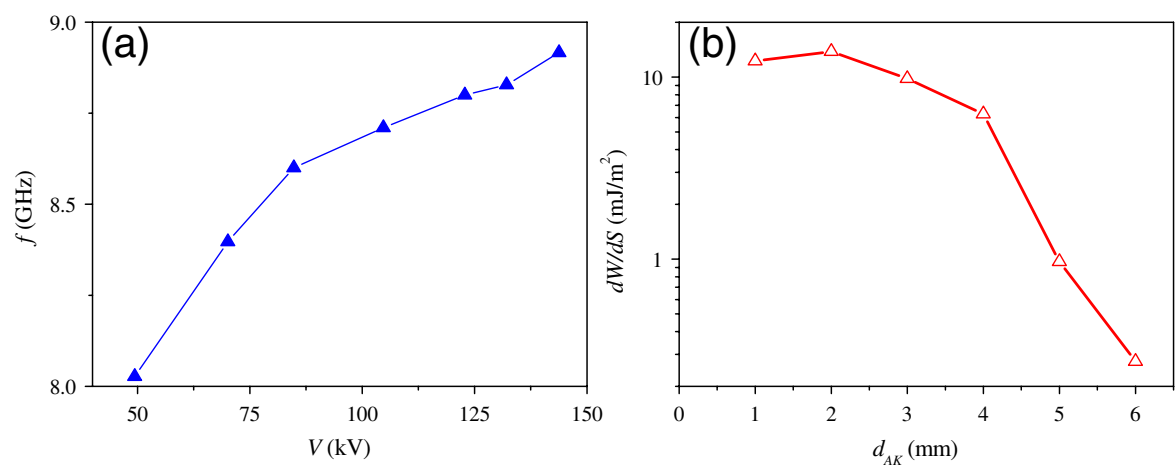

FIG. 4. (Color) (a) Second harmonic frequency vs peak voltage. (b) X-band horn energy/area vs $d_{\mathrm{AK}}$.

forward direction. For the second harmonic, with the mirror placed at the ideal angle, we found $5 \pm 1 \mathrm{~mJ}$ in a circular cone of $15^{\circ}$ half-angle. For the third harmonic, we obtained $0.04 \pm 0.01 \mathrm{~mJ}$. The analysis of the S-band horn, which sees the evanescent wave, showed a poorly defined emission pattern, consistent with the nondirectional character of this radiation. We obtained a crude estimate of the energy in a $22^{\circ}$ cone, $10 \pm 3 \mathrm{~mJ}$. Since the total beam energy in a pulse is $3 \mathrm{~J}$, the efficiency for the second harmonic is approximately $0.15 \%$. Using the time signal in the $\mathrm{X}$-band horn, we estimated the temporal gain, $\operatorname{Im} \omega=(1.3 \pm 0.3) \times 10^{9} \mathrm{~s}^{-1}$. Since we were unable to substantially vary the current $I$, we cannot say how $\operatorname{Im} \omega$ depends on it. When we look in detail at Fig. 2(a), we see that the signal rises from noise to saturation in approximately $4 \mathrm{~ns}$. These results may be compared to our 2D simulations of Ref. [14], where we found a gain of order $2 \times 10^{9} \mathrm{~s}^{-1}$ for the current used here.

\section{CONCLUSION}

In preparation for this experiment, two of us performed simulations using the code MAGIC in both 2D and 3D versions. The aim was to see whether such simulations would justify our claim to validate the $2 \mathrm{D} \mathrm{AB}$ theory. We found that both simulations agreed rather well in their predictions concerning the evanescent wave and the current bunching. Some of our experimental observations are in reasonable accord with these simulations: (i) The measured value of the magnetic field at the end of the groove agrees rather well with what our 3D simulation predicts. (ii) The simulations show that energy of the beam in the interaction region is less than the nominal emitted energy because of space-charge effects. This accounts for some of the discrepancy between the applied voltage and the energy deduced from the frequency of the radiation via the $A B$ dispersion relation. (iii) The current rise time from zero to half the maximum value occurs in about $17 \mathrm{~ns}$, which is much longer than the time in which the radiation reaches its saturated value. The important question of the value of the start current cannot be addressed with the data we have, but we plan to measure it in a modified setup where the cathode can be shielded so as to reduce the applied field on its surface without decreasing the voltage. In this way we hope to vary the current so as to establish experimentally the start current, along with the gain as a function of current. Our earlier 2D simulation suggested a start current in the range of 10-15 A/m. In our experiment we used a much higher value, $\approx 1800 \mathrm{~A} / \mathrm{m}$.

We consider that these results validate some major aspects of the 2D theory of Andrews and Brau; in particular, the observed frequency is quite close to that predicted by their 2D dispersion relation. As was shown in Ref. [19], a 3D simulation of the dispersion relation for our grating is practically indistinguishable from the $2 \mathrm{D}$ calculation. Since the $2 \mathrm{D} A B$ theory has no intrinsic scale, any frequency could in principle be produced with an appropriately scaled setup. Of course, when the scale is reduced, so as to produce $\mathrm{THz}$ radiation, the constraints on beam size become quite difficult to satisfy. Nevertheless, provided sufficiently good beam quality can be achieved, the goal of a tabletop $\mathrm{THz}$ source should be attainable.

[1] S. J. Smith and E. M. Purcell, Phys. Rev. 92, 1069 (1953).

[2] J. Urata, M. Goldstein, M. F. Kimmitt, A. Naumov, C. Platt, and J. E. Walsh, Phys. Rev. Lett. 80, 516 (1998).

[3] A. Bakhtyari, J. E. Walsh, and J. H. Brownell, Phys. Rev. E 65, 066503 (2002).

[4] K. J. Woods, J. E. Walsh, R. E. Stoner, H. G. Kirk, and R. C. Fernow, Phys. Rev. Lett. 74, 3808 (1995).

[5] O. Haeberlé, P. Rullhusen, N. Maene, and W. Mondelaers, Phys. Rev. E 60, 6214 (1999).

[6] G. Doucas, M. F. Kimmitt, A. Doria, G. P. Gallerano, E. Giovenale, G. Messina, H.L. Andrews, and J.H. Brownell, Phys. Rev. ST Accel. Beams 5, 072802 (2002).

[7] G. Kube et al., Phys. Rev. E 65, 056501 (2002).

[8] S. Kesar, R. A. March, and R. J. Temkin, Phys. Rev. ST Accel. Beams 9, 022801 (2006).

[9] V. Blackmore, G. Doucas, C. Perry, and M. F. Kimmitt, Nucl. Instrum. Methods Phys. Res., Sect. B 266, 3803 (2008).

[10] C. S. Liu and V. K. Tripathi, IEEE J. Quantum Electron. 35, 1386 (1999). 
[11] V.P. Shestopalov, Generators of Diffraction Radiation (Naukova Dumka, Kiev, 1991), in Russian.

[12] H.L. Andrews and C.A. Brau, Phys. Rev. ST Accel. Beams 7, 070701 (2004).

[13] L. Andrews, C. H. Boulware, C. A. Brau, and J. D. Jarvis, Phys. Rev. ST Accel. Beams 8, 050703 (2005); 8, 110702 (2005).

[14] J. T. Donohue and J. Gardelle, Phys. Rev. ST Accel. Beams 8, 060702 (2005).

[15] B. Goplen, L. Ludeking, D. Smithe, and G. Warren, Comput. Phys. Commun. 87, 54 (1995).

[16] D. Li, Z. Yang, K. Imasaki, and Gun-sik Park, Phys. Rev. ST Accel. Beams 9, 040701 (2006).

[17] Vinit Kumar and Kwang-Je Kim, Phys. Rev. E 73, 026501 (2006).
[18] H. L. Andrews, C. A. Brau, J. D. Jarvis, C. F. Guertin, A. O'Donnell, B. Durant, T. H. Lowell, and M. R. Mross, Phys. Rev. ST Accel. Beams 12, 080703 (2009).

[19] H. L. Andrews, C. A. Brau, and J. D. Jarvis, J. Appl. Phys. 105, 024904 (2009).

[20] Kwang-Je Kim and Vinit Kumar, Phys. Rev. ST Accel. Beams 10, 080702 (2007).

[21] J. Gardelle and J.T. Donohue, IEEE Trans. Electron Devices 56, 769 (2009).

[22] J.D. Jackson, Classical Electrodynamics (John Wiley \& Sons, New York, 1975), 2nd ed., p. 583.

[23] B. K. Skrynnik, V.K. Korneyenkov, and M. Yu. Demchenko, Telecommun. Radio Eng. 55, 170 (2001). 